\title{
Dendron Arrays for the Force-Based Detection of DNA Hybridization Events
}

\author{
Yu Jin Jung, ${ }^{1}$ Bong Jin Hong, ${ }^{1}$ Wenke Zhang, ${ }^{2}$ Saul J. B. Tendler, ${ }^{2}$ Philip M. Williams, ${ }^{2}$ \\ Stephanie Allen ${ }^{2 *}$ and Joon Won Park ${ }^{1^{*}}$
}

Center for Integrated Molecular Systems, Department of Chemistry, Pohang University of Science and Technology, San 31 Hyoja-dong, Pohang, 790-784, Korea

Laboratory of Biophysics and Surface Analysis, School of Pharmacy, The University of Nottingham, Nottingham NG7 2RD, U.K.

\section{Supporting Information}

\section{(1) The influence of base-layer chemistry and dendron generation}

Either glycidylpropyldiethoxymethylsilane (or GPDES) or N-(3-(triethoxysilyl)propyl)O-polyethyleneoxide urethane (or TPU) was employed to generate a base layer, and the dendron (9-anthrylmethyl $\mathrm{N}-(\{[\operatorname{tris}(\{2-[(\{\operatorname{tris}[(2-$ carboxyethoxy $)$ methyl $]$ methyl $\}$ amino $)$ carbonyl]ethoxy\}methyl)methyl]amino\} carbonyl) propylcarbamate) (or 9-acid) 
immobilized onto them. Previously, the mesospacing between the dendrons on the GPDESmodified surface was found to be approximately $32 \AA{ }^{28}{ }^{28}$ For TPU modified substrates, the magnitude of the absorption peak observed at $257 \mathrm{~nm}$ arising from the anthryl moiety of the pristine dendron (see ref. 27 for method) was approximately one half of that observed for the GPDES substrates, suggesting that the spacing of the dendrons on these substrates is less dense and greater than $32 \AA$.

To understand effect of the increased spacing afforded by the TPU base layer on the interactions of oligonucleotide functionalized dendron surfaces, parallel sets of forcemeasurements were recorded on substrates employing both types of attachment chemistry. Importantly, since it is only possible to determine AFM spring constants with $\sim 10 \%$ certainty, to eliminate variation in the data due to such factors these initial comparative experiments were also performed with the same tip (functionalized with 9-acid/TPU and 30-base (30 mer) oligonucleotide sequence).

Measurements were first obtained for a 9-acid/GPDES substrate functionalized with the 30 mer oligonucleotide sequence complementary to that immobilized on the tip. The obtained distribution of unbinding forces was broad with several maxima, suggesting that 
the recorded forces were due to the rupture of many multiples of, rather than single, oligonucleotide interactions (Figure S1(a)). Consistent with this behaviour the retract traces of many of the obtained force-measurements also displayed multiple 'pull-off' events. In contrast, when the same DNA sequence was immobilized on the 9-acid/TPU surface, the distribution of unbinding forces (Figure S1(b)) was more narrow, with one maximum at 38 $\mathrm{pN}$, and all of the obtained force measurements displayed clean single pull-off events. While such force measurements and the disappearance of the higher force maxima are consistent with the measurement of a reduced number of oligonucleotide interactions for the less dense TPU surface, the fact that adhesion events were observed in greater than $90 \%$ of measurements indicates that they still correspond to the rupture of multiple interactions. ${ }^{34}$ Further increases in oligonucleotide spacing, as achieved through the use of substrates functionalized with the third generation dendron (27-acid), resulted in a reduction in the frequency of observing an adhesion event (to approximately $50 \%$ ), although the maxima of force distribution remained at the approximately the same value. 
(a)

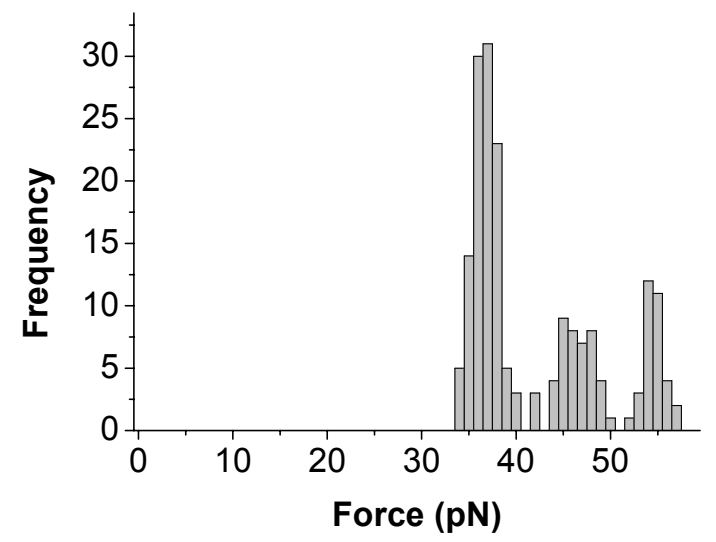

(b)

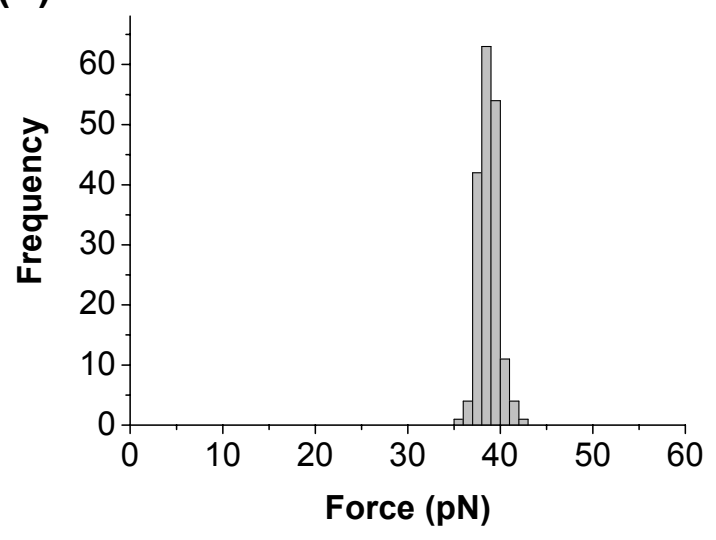

Figure S1. Distributions of adhesion forces (recorded with a retract velocity of $0.1 \mu \mathrm{m} / \mathrm{s}$ ) obtained for 30 mer oligonucleotide functionalized dendron surfaces employing the (a) GPDES and (b) TPU base-layer chemistry respectively. 
(2) Single and double base mismatch data

(a)

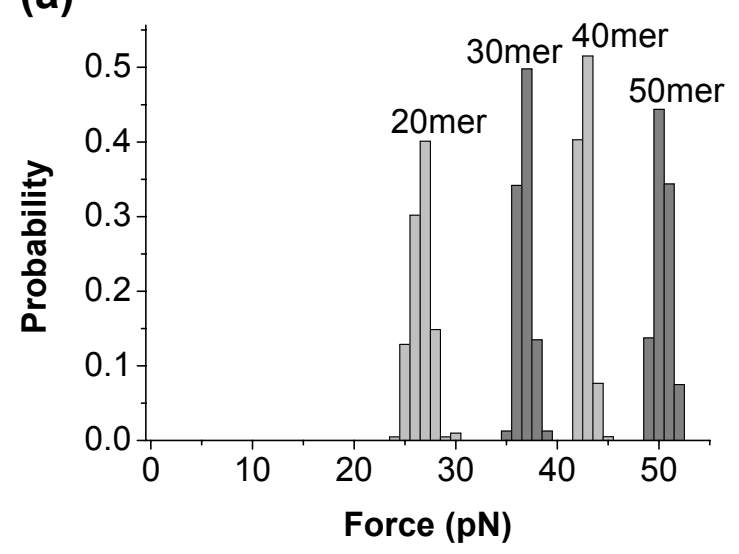

(b)

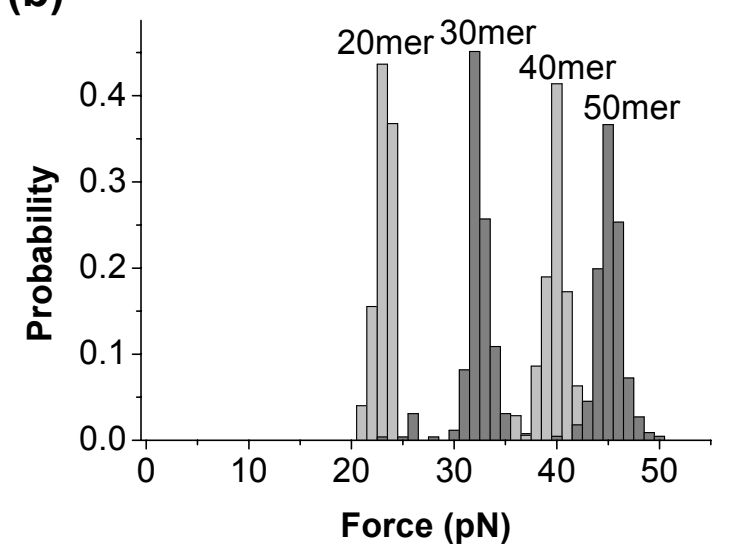

Figure S2. Distribution of the attractive forces recorded at $0.1 \mu \mathrm{ms}^{-1}$ for all of the (a) single base and (b) double base mismatch sequences detailed in Table 1. The y-axis reflects the probability of observing a force of particular magnitude within each distribution. 